\title{
A New Approach for Safe Tube Thoracostomy Insertion: An Objective and Subjective Comparison versus Established Techniques
}

\author{
Alex Doyle, Thomas White, Anna Hutton, Karen Mcguire, Parvez Moondi", Peter Young \\ Department of Critical Care, Queen Elizabeth Hospital, Kings Lynn, Norfolk, UK. \\ Email: ajdoyle@gmail.com, thomas.white@qehkl.nhs.uk, annahutton@doctors.org.uk, karen.mcguire@qehkl.nhs.uk, \\ parvez.moondi@qehkl.nhs.uk, peter.young@qehkl.nhs.uk
}

Received November $20^{\text {th }}, 2013$; revised December $18^{\text {th }}, 2013$; accepted January $15^{\text {th }}, 2014$

Copyright (C) 2014 Alex Doyle et al. This is an open access article distributed under the Creative Commons Attribution License, which permits unrestricted use, distribution, and reproduction in any medium, provided the original work is properly cited. In accordance of the Creative Commons Attribution License all Copyrights @ 2014 are reserved for SCIRP and the owner of the intellectual property Alex Doyle et al. All Copyright (C 2014 are guarded by law and by SCIRP as a guardian.

\section{ABSTRACT}

Introduction: Tube thoracostomy is an invasive procedure, which may result in life-threatening injury to major organs and blood vessels. We propose a new approach for inserting tube thoracostomies to improve the safety of this procedure, termed guided blunt dissection. In this article, we compared the safety of this new approach with traditional blunt dissection and two commercially available Seldinger tube thoracostomy kits in an ex vivo model. Methods: We recruited 32 clinicians from a variety of medical specialties with a range of experience in performing tube thoracostomy. Each clinician was required to perform tube thoracostomy using all four approaches in a randomised order. Objectively, each insertion was categorised as "safe" if the lung remained intact and "unsafe" if the lung deflated. Subjectively, participants were asked to rank each approach in order of perceived safety on a four-point scale. Statistical analysis was performed using a Fisher's exact test. Results: Objectively, guided blunt dissection was significantly safer than both Seldinger approaches $(p<0.0001)$, but not traditional blunt dissection $(p=0.71)$. Subjectively, none of the approaches were felt to be superior. Conclusions: These data support the conclusions that, in this ex vivo model, the new guided blunt dissection approach provided a safe method for tube thoracostomy. Guided blunt dissection produced less lung deflations relative to competing methods, certainly when compared objectively to Seldinger techniques. Of note, the Seldinger approaches were perceived by the participants to be as safe despite there being an increased incidence of lung injury associated with their use in this model. This indicates that it was not always possible for the clinician to determine when lung injury had occurred. This potential for lung injury when using Seldinger approaches for tube thoracostomy should be emphasised.

\section{KEYWORDS}

Thoracostomy; Chest Tube; Complications; Guided Blunt Dissection

\section{Introduction}

Tube thoracostomies are used in many different settings and clinicians from many acute specialties need to be capable of their safe placement, even if they do not perform the procedure regularly. However, serious complications are possible, including life-threatening injury to major organs (heart, lung, diaphragm and intra-abdominal or-

"Corresponding author. gans) and blood vessels, even when performed by experienced operators $[1,2]$.

Traditionally, tube thoracostomies were inserted along a tract made by blunt dissection with forceps. Before placing the tubing, the tract was explored with a finger to ensure that there were no underlying structures adherent to the pleura $[3,4]$. Disadvantages of this technique include possible injury to the clinician when exploring the tract with their fingers from a rib fracture and loss of the 
tract before placing the tubing. The latter disadvantage may be a particular problem in obese patients.

Recently, Seldinger tube thoracostomy kits have become increasingly popular [5,6]. The pleural cavity is identified by aspirating air or fluid through a needle, which is blindly introduced into the thoracic cavity. A wire is then passed down the needle and a series of dilators is used to open up the tract before inserting the tubing. Advantages of this technique include avoidance of possible injury to the clinician, avoidance of a large incision to the skin and maintenance of the tract using a wire. A major disadvantage of this technique is the blind introduction of the needle, which may puncture underlying organs, blood vessels and body cavities, causing significant patient morbidity and mortality [2].

We propose a new approach, termed guided blunt dissection, by inserting tube thoracostomies, which we have designed to improve the safety of this procedure. It combines the advantages of traditional blunt dissection and more recent Seldinger techniques, but avoids the disadvantages of both. Furthermore, it is easy to use because it relies on the same familiar principles as the current approaches. It utilises a novel set of blunt dissection forceps, which contain an integral wire channel (Figure 1). Using this technique, blunt dissection can be performed using the forceps. Once the thoracic cavity is reached, the tract can be maintained by passing a wire along the channel in the forceps. Finally, the tube can be inserted directly or one or more Seldinger dilators are used to open up the tract before inserting the tube. If necessary, intrathoracic placement can be confirmed by syringe aspiration of the forceps channel prior to passing the wire.

In this report, we objectively and subjectively compared the safety of this new approach with traditional blunt dissection and two commercially-available Seldinger tube thoracostomy sets using an ex vivo model.

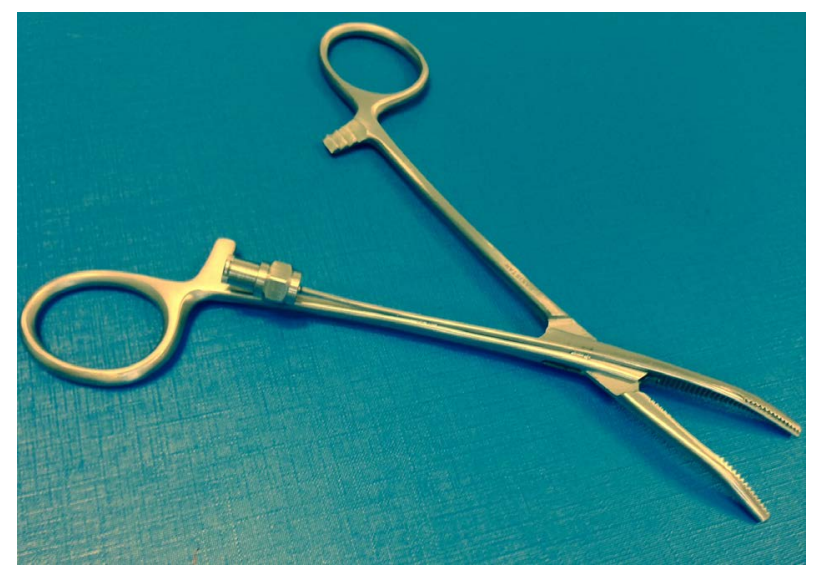

Figure 1. A photograph of the novel guided blunt dissection forceps.

\section{Methods}

\subsection{Ethics}

Approval for the project was granted from the regional ethical committee prior to commencement of the study. Written informed consent was obtained from all participants.

\subsection{Participants}

All participants were medically qualified and declared that they would be expected to perform a tube thoracostomy unsupervised as part of their everyday duties. None had prior knowledge that the study was about to take place.

\subsection{Equipment}

All equipment was used according to the manufacturers' instructions. The two Seldinger tube thoracostomy kits were: Thal-Quick (model number: C-TQTS-1800, 18 French gauge, Cook Medical Inc, Bloomington, IN, USA) and Rocket Seldinger (model number: RK 54544-18-PK, 18 French gauge, Rocket Medical, Tyne \& Wear, UK). Two different commercial Seldinger kits were used as they contained differing introducer needles and it was felt that this could result in a variation in lung puncture rates.

\subsection{Randomization}

A closed envelope technique was used to randomly allocate the order that participants performed all four approaches.

\subsection{Ex Vivo Model}

A pilot study was performed to facilitate the model design. The response of porcine lung and a number of synthetic model lungs to penetrating injury was compared. A latex model lung exhibiting behaviour most closely resembling that of porcine lung was chosen for the study. This consisted of a latex balloon inflated and covered with a mepore dressing (Molnlycke Healthcare, GA, USA). The mepore dressing was used as it was found to reduce the noise of balloon puncture and slow deflation. This replicated actual lung more closely and removed the audible cue for the participants if simulated lung injury occurred during a procedure. The surface area of the model lung in contact with the thoracic wall was coated in a water-soluble gel (K-Y gel, Johnson and Johnson, NJ, USA). A sheep's thorax was used to simulate the thoracic wall. The model was secured on a desk and draped in surgical dressings, exposing an operative field.

We specifically designed the model to reflect those instances where the likelihood of lung injury was high, 
namely where lung tissue was adjacent to the parietal pleura.

\subsection{Procedure}

For each participant, an investigator delivered a standardized introduction and demonstration of how to perform a tube thoracostomy using each approach in accordance with accepted medical practice $[3,4]$. The demonstration time was approximately 5 minutes per approach. Next, each participant was asked to perform that particular approach utilizing the ex vivo model. The same format was used for each of the four approaches. A closed envelope technique was used to randomly allocate the order of approaches. For each approach the correct equipment was placed next to the model.

\subsection{Objective Comparison}

The pilot study indicated that the model lung would puncture and slowly deflate when injured during the procedure. Therefore, lung injury was defined as any episode of lung deflation that occurred during the procedure. Each insertion was categorised as "safe" if the lung remained intact, and "unsafe" if the lung deflated.

\subsection{Subjective Comparison}

After the participant had completed all four procedures, we asked them to rank each technique in order of safety on a four-point scale. A score of 4 was given to the approach deemed to be the most safe and 1 given to that deemed the least safe. The scores for each approach were summed and the total score used to provide a subjective estimate of the safety of each approach.

\subsection{Sample Size Calculation}

There is limited data that describes the incidence of lung injury following tube thoracostomy insertion for a pneumothorax. Seeing that we were testing a model where the likelihood of lung injury was high, we estimated the incidence of lung injury would be high at 0.35 using established techniques and much lower at 0.05 using guided blunt dissection. This revealed that we required a minimum sample size of 30 assuming an $\alpha$ level of 0.05 and a $\beta$ level of 0.5 to demonstrate a significant difference between approaches.

\subsection{Statistical Analysis}

Forthe objective comparison statistical analysis was performed using a Fisher's exact test and a Bonferroni correction was applied. A $p$ value $<0.05$ was considered statistically significant. For the subjective analysis the technique with the highest score was considered safest using this analysis.

\section{Results}

We recruited 32 participants from a variety of medical specialties with a range of clinical experience and experience in performing tube thoracostomy (Table 1). Objectively, the guided blunt dissection had significantly fewer deflations of the test lung than both Seldinger approaches (Table 2). Subjectively, none of the approaches were felt to be superior (Table 3).

Table 1. The range of medical specialties and experience of participants.

\begin{tabular}{|c|c|}
\hline Criteria & Number of participants \\
\hline \multicolumn{2}{|c|}{ Medical specialty } \\
\hline Medicine & 10 \\
\hline Surgery & 11 \\
\hline Anesthesia & 11 \\
\hline \multicolumn{2}{|c|}{ Range of clinical experience (years) } \\
\hline $0-4$ & 12 \\
\hline $5-10$ & 8 \\
\hline$>10$ & 12 \\
\hline \multicolumn{2}{|c|}{ Number of times procedure performed previously } \\
\hline $0-4$ & 10 \\
\hline $5-10$ & 5 \\
\hline$>10$ & 17 \\
\hline
\end{tabular}

Table 2. Objective comparison of the safety of each technique.

\begin{tabular}{cccc}
\hline Technique & Safe & Unsafe & $\boldsymbol{p}$ value \\
\hline Guided blunt dissection & 27 & $5(16 \%)$ & - \\
Blunt surgical dissection & 29 & $3(9.4 \%)$ & 0.71 \\
Thal-Quick Seldinger & 2 & $30(93.75 \%)$ & $<0.0001$ \\
Rocket Seldinger & 10 & $22(68.75 \%)$ & $<0.0001$ \\
\hline
\end{tabular}

Table 3. Subjective comparison of the safety of each technique (a high score signifies the most safe technique).

\begin{tabular}{cc}
\hline Technique & Total score \\
\hline Guided blunt dissection & 85 \\
Rocket Seldinger & 84 \\
Blunt surgical dissection & 82 \\
Thal-Quick Seldinger & 69 \\
\hline
\end{tabular}




\section{Discussion}

Blunt dissection and Seldinger tube thoracostomy sets are established in the management of pleural disease. However, serious harm is possible using either approach, even when performed by experienced clinicians. Undoubtedly this occurs because in some cases the procedure is more difficult to perform than in others. For example, it may be difficult to identify the "safe triangle" in a morbidly obese patient $[3,4]$. The "safe triangle" is a region bordered by the lateral border of pectoralis major, a horizontal line inferior to the axilla, the anterior border of latissimus dorsi and a horizontal line superior to the nipple. Furthermore, although tube thoracostomy is a common procedure, it may be necessary for a clinician with limited experience of the technique to perform it.

We designed the guided blunt dissection forceps in response to concerns we had with both traditional blunt dissection and Seldinger tube thoracostomy techniques, regarding the safety of the patient and the clinician. This new approach seeks to avoid injury to the clinician when exploring the tract with their fingers, and potential injury to the patient from blind needle insertion. Our report demonstrates that guided blunt dissection provides a safe approach for inserting tube thoracostomies in an ex vivo model when compared with traditional techniques.

Previously, it has been demonstrated that lung trauma was significantly more likely using Seldinger kits to insert a tube thoracostomy compared to traditional blunt dissection [7]. Our study confirms and extends these findings. We observed that all lung perforations using the Seldinger tube thoracostomy sets occurred during blind needle insertion, highlighting the dangers of this approach. The discrepancy between the subjective and objective comparison was striking. For example, the Rocket Seldinger approach was rated almost identically safe as guided and traditional blunt dissection subjectively (subjective scores of 84 versus 85 and 82) and yet objectively, the Rocket Seldinger approach produced more lung deflations when compared with other kits (69\% versus 16\% and 9.4\%). This suggests that it was not clear to the clinician when lung injury occurred using a Seldinger tube thoracostomy kit. These kits were falsely perceived to be as safe and there was a lack of insight into the dangers of Seldinger tube thoracostomy among our participants. This highlights the need to consider the perceived safety of these kits and what can be done to make them safer.

In clinical practice, the safety of blind needle insertion can be improved by using ultrasound to guide needle placement $[8,9]$. However, ultrasound guided tube thoracostomy insertion is a specialist skill, which requires training. The availability of an ultrasound machine or trained personnel cannot always be guaranteed. In the United Kingdom, an ultrasonographer often directs the point of needle insertion and the procedure is performed on a separate occasion by the clinician. We caution against this approach because there is a risk that the fluid and organs can shift in the time between the ultrasound examination and the operative procedure [9]. In addition, ultrasound cannot be used to reliably distinguish between lung and the air-filled pleural space in the treatment of a pneumothorax.

We recommend that blunt dissection should be used in the first instance. In this study, we did not demonstrate a significant difference in safety between guided blunt dissection and traditional blunt dissection. However, one disadvantage of traditional blunt dissection is injury to the clinician from rib fractures. This is reduced using the guided technique which makes it a desirable option.

There were limitations to this study. This is an ex vivo model rather than a clinical study. However, simulation of practical scenarios is now commonly used for testing new medical devices. Our model may have demonstrated a higher than expected rate of lung puncture because the lung was adjacent to the chest wall. No attempt was made to simulate pneumothorax or pleural effusion. There were lung perforations using blunt surgical dissection and guided blunt dissection in our study. These occurred when participants used a scalpel to incise the skin prior to dissection of the tract, rather than during the blunt dissection itself. Whilst these injuries serve to highlight that injury can occur when using these approaches, they may also reflect a limitation of our ex vivo model as the sheep thorax used had limited adipose tissue. It is likely that guided blunt dissection forceps will be particularly useful in the placement of tube thoracostomy in obese patients, where it can be difficult to maintain the tract prior to tube insertion. However, it was not possible to test for this using our ex vivo model. There was also the potential for bias because it is impossible to blind the participants to the device being used.

These data support the conclusion that, in this ex vivo model, the new guided blunt dissection approach provided a safe method for tube thoracostomy. Guided blunt dissection produced fewer lung deflations relative to competing methods, particularly when compared objectively to Seldinger techniques. Of note, the Seldinger approaches were perceived by the participants to be as safe despite there being an increased incidence of lung injury associated with their use in this model. This potential for lung injury when using Seldinger approaches for tube thoracostomy should be emphasised

\section{Acknowledgements}

TW and AD conducted the study, drafted the write up study and are the lead authors of the submitted manuscript. AH undertook analysis and interpretation of data 
and critically revised the manuscript. KM procured the equipment and conducted the study. PM and PY devised and supervised the study and critically revised the manuscript. All authors have given final approval for the version to be published.

\section{Competing Interests}

Drs Moondi and Young hold the patent for the guided blunt dissection forceps. The remaining authors do not declare any conflict of interest.

\section{REFERENCES}

[1] K. S. Miller and S. A. Sahn, "Chest Tubes. Indications, Technique, Management and Complications," Chest, Vol. 91, No. 2, 1987, pp. 258-264. http://dx.doi.org/10.1378/chest.91.2.258

[2] A. Harris, B. R. O’Driscoll and P. M. Turkington, "Survey of Major Complications of Intercostal Chest Drain Insertion in the UK," Postgraduate Medical Journal, Vol. 86, No. 1012, 2010, pp. 68-72. http://dx.doi.org/10.1136/pgmj.2009.087759

[3] S. P. Dev, B. Nascimiento Jr., C. Simone and V. Chien. "Videos in Clinical Medicine. Chest-Tube Insertion," New England Journal of Medicine, Vol. 357, No. 15, 2007, p. e15. http://dx.doi.org/10.1056/NEJMvcm071974
[4] D. Laws, E. Neville and J. Duffy, "BTS Guidelines for the Insertion of a Chest Drain,” Thorax, Vol. 58, Suppl. 2, 2003, pp. ii53-ii59. http://dx.doi.org/10.1136/thorax.58.suppl_2.ii53

[5] A. Horsley, L. Jones, J. White and M. Henry, "Efficacy and Complications of Small-Bore, Wire-Guided Chest Drains," Chest, Vol. 130, No. 6, 2006, pp. 1857-1863. http://dx.doi.org/10.1378/chest.130.6.1857

[6] H. E. Davies, S. Merchant and A. McGown, "A Study of the Complications of Small Bore 'Seldinger' Intercostal Chest Drains,” Respirology, Vol. 13, No. 4, 2008, pp. 603-607.

http://dx.doi.org/10.1111/j.1440-1843.2008.01296.x

[7] N. A. Collop, S. Kim and S. A. Sahn, "Analysis of Tube Thoracostomy Performed by Pulmonologists at a Teaching Hospital,” Chest, Vol. 112, No. 3, 1997, pp. 709-713. http://dx.doi.org/10.1378/chest.112.3.709

[8] A. H. Diacon, M. H. Brutsche and M. Soler, "Accuracy of Pleural Puncture Sites: A Prospective Comparison of Clinical Examination with Ultrasound," Chest, Vol. 123, No. 2, 2003, pp. 436-441. http://dx.doi.org/10.1378/chest.123.2.436

[9] T. Havelock, R. Teoh, D. Laws and F. Gleeson, "Pleural Procedures and Thoracic Ultrasound: British Thoracic Society Pleural Disease Guideline 2010,” Thorax, Vol. 65, Suppl. 2, 2010, pp. i61-i76. http://dx.doi.org/10.1136/thx.2010.137026 